Jun Akanuma • Kae Muraki • Hirofumi Komaki

Ikuya Nonaka • Yu-ichi Goto

\title{
Two pathogenic point mutations exist in the authentic mitochondrial genome, not in the nuclear pseudogene
}

Received: August 2, 2000 / Accepted: August 30, 2000

\begin{abstract}
Technical advancements in molecular genetics have shown various mitochondrial DNA (mtDNA) abnormalities in patients with mitochondrial myopathies. Recently, it has been revealed that, in these patients, the nuclear DNA carries sequences similar to those of the mtDNA (nuclear pseudogene) and it has several point mutations previously reported to be pathogenic. We verified the existence of the T3250C and T3291C mutations, which we have found in patients with mitochondrial myopathy, in the authentic mitochondrial genome. A long polymerase chain reaction provides a powerful tool for avoiding nuclear pseudogene amplification and for ruling out ambiguity in the detection of the mutation for diagnosis.
\end{abstract}

Key words Mitochondrial DNA $\cdot$ Pseudogene $\cdot$ Long PCR - Point mutations · Rho-0 cell

\section{Introduction}

Human mitochondrial DNA (mtDNA) is a 16,569-bp, circular, double-stranded molecule that encodes 13 protein subunits of four respiratory chain enzyme complexes, 22 tRNAs, and 2 rRNAs. Current technical advances in molecular genetics have led to various mtDNA abnormalities being reported in patients with mitochondrial myopathies.

J. Akanuma $\cdot$ K. Muraki $\cdot$ H. Komaki $\cdot$ Y. Goto $(\square)$

Department of Mental Retardation and Birth Defect Research,

National Institute of Neuroscience, National Center of Neurology

and Psychiatry, 4-1-1 Ogawahigashi, Kodaira, Tokyo 187-8502, Japan

Tel. +81-42-346-1713; Fax +81-42-346-1743

e-mail: goto@ncnp.go.jp

I. Nonaka

Department of Ultrastructural Research, National Institute of Neuroscience, National Center of Neurology and Psychiatry,

Kodaira, Tokyo, Japan
The relative ease of whole mtDNA sequencing has led to more than 50 pathogenic point mutations and several kinds of rearrangements having been detected in the past 11 years (DiMauro et al. 1998). Recently, several mtDNA point mutations were identified in some tumors and these mutations were assumed to be dominant (Fliss et al. 2000). This result means the damage to mtDNA occurred not only in mitochondrial myopathies but also in neoplasms.

We reported an A3243G substitution in mitochondrial myopathey, encephalopathy, lactic acidosis, and stroke-like episodes (MELAS) (Goto et al. 1990) following T3250C and T3291C mutations, all of which are in the mitochondrial tRNA-Leu (UUR) gene (Goto et al. 1992; 1994). Subsequent studies detected at least 11 different pathogenic point mutations and a single base deletion in this region (MITOMAP; http://infinity.gen.emory.edu/mitomap.html). The mitochondrial tRNA-Leu (UUR) gene is therefore important for the genetic diagnosis of mitochondrial myopathy.

Recently, Davis et al. (1997), reported that specific point mutations in the $C O I$ and $C O I I$ genes of mtDNA cause the cytochrome c oxidase (COX) defect in Alzheimer disease, but subsequent studies showed that the point mutations were located on the nuclear DNA, which carries nuclear pseudogenes highly similar to those of mitochondrial $C O X$ genes (Hirano et al. 1997; Davis et al. 1998). Taylor and coworkers found a novel mitochondrial tRNA-Leu (UUR)/ ND1 pseudogene in the nuclear DNA which had several mutations, including T3250C and T3291C (Taylor et al. 1998). They pointed out that the pseudogene would be amplified by polymerase chain reactions (PCRs) used for diagnosis and research, thereby modifying the results of both heteroplasmic base substitutions. Parfait et al. (1998) also observed co-amplification of nuclear pseudogene(s) in nucleotide positions 3130 to 3423 , using two different cell lines lacking mtDNA (rho-0 cell lines).

The purpose of our study was to verify the existence of previously reported point mutations and to investigate the effects of the nuclear pseudogene of mtDNA on genetic diagnosis. We employed the long PCR method to minimize any adverse effects of the nuclear pseudogene. 


\section{Materials and methods}

Cell culture. HeLa cells with mtDNA and HeLa-derived cell lines lacking mtDNA (rho-0 cell) were cultured as described elsewhere (Hayashi et al. 1991). The absence of mtDNA in the rho- 0 cells had previously been confirmed by Southern blot analysis (Hayashi et al. 1991).

Patients. Elsewhere we reported a T-to-C transition mutation at nucleotide position 3250 in a family with mitochondrial myopathy and a T-to-C point mutation at nucleotide pair 3291 in a Japanese patient with MELAS (Goto et al. 1992; 1994).

Extraction of DNA and mtDNA sequence analysis. DNA was extracted from muscle samples taken from the patients with the various point mutations and from the HeLa and rho-0 cells by phenol-chloroform extraction and ethanol precipitation. We constructed 12 sets of primers for amplifying the DNA fragments, and 99 sequence primers for sequencing whole mtDNA (Nishino et al. 1996). PCR products were purified in MicroSpin S-400HR columns (Pharmacia Biotech, NJ, USA), and cycle sequencing was done with a BigDye Terminator Cycle Sequencing Ready Reaction Kit (Applied Biosystems, Foster City, CA, USA) as recommended by the manufacturer. Excess ddNTP was removed by Microspin S-200 columns (Pharmacia Biotech). Samples were electrophoresed through a 5\% Long Ranger gel (FMC BioProducts, Rockland, USA) in a model 377 automatic DNA sequencer (Applied Biosystems).

Detection of T3250C and T3291C mutations. We previously designed mismatched primers to detect these mutations (Goto et al. 1992; 1994). To identify T3250C, we amplified the 199-bp fragment and performed endonuclease NaeI digestion. If the pragment had the T3250C mutation, cleaved fragments of 177 and $22 \mathrm{bp}$ could be detected. To detect the T3291C mutation, the 309-bp fragment with the mismatched and reverse primers was digested by BamHI. If the mutation was present, cleaved fragments of 280 and $29 \mathrm{bp}$ were obtained. Each fragment was detected in a $4 \%$ agarose gel (NuSieve 3:1 Agarose; FMC Bio products) stained with ethidium bromide.

Long PCR. The long PCR reactions were conducted with two sets of primers: P329/P318 for short fragments and P3024/P3025 for large ones. The primer sequences were: P329, 5'-GAATCGGAGGACAACCAGTA-3'; P318, 5'GCGGGAGAAGTAGATTGAAG-3'; P3024, 5' -TTCCA TCCACCCTCCTCTCCCTAGG-3'; and P3025, 5' -GGGA TATTGATTTCACGGAGGATGG-3'. The reaction mixture consisted of $0.2 \mathrm{mM}$ of each dNTP, 1.0 units of Takara Ex Taq (Takara, Kyoto, Japan), $1 \times$ Ex Taq Buffer, $0.2 \mu \mathrm{M}$ of each primer, and the extracted genomic DNA (total volume, $50 \mu \mathrm{l})$. PCR amplification was done with a GeneAmp PCR System 2400 (Applied Biosystems) with 30 cycles of denaturation at $94^{\circ} \mathrm{C}$ for $20 \mathrm{~s}$, annealing at $60^{\circ} \mathrm{C}$ for $20 \mathrm{~s}$, and extension at $72^{\circ} \mathrm{C}$ for $5 \mathrm{~min}$. Amplified PCR fragments were separated electrophoretically in a $1.0 \%$ agarose gel (Agarose S; NipponGene, Tokyo, Japan) after ethidium bromide staining. To sequence the tRNA-Leu (UUR) region, the PCR products were purified with a QIAEX II Gel Extraction Kit (Qiagen, Chatsworth, CA, USA) before cycle sequencing.

\section{Results}

Nuclear pseudogene sequence similar to mitochondrial tRNA-Leu (UUR)

PCR amplification was done with DNA extracted from HeLa and rho- 0 cells, using 12 overlapping primer sets for mtDNA. Five fragments, 1, 2, 4, 6, and 9, were clearly amplified with the rho- 0 cell DNA, indicative of the presence of the nuclear pseudogene (Fig. 1). PCR products
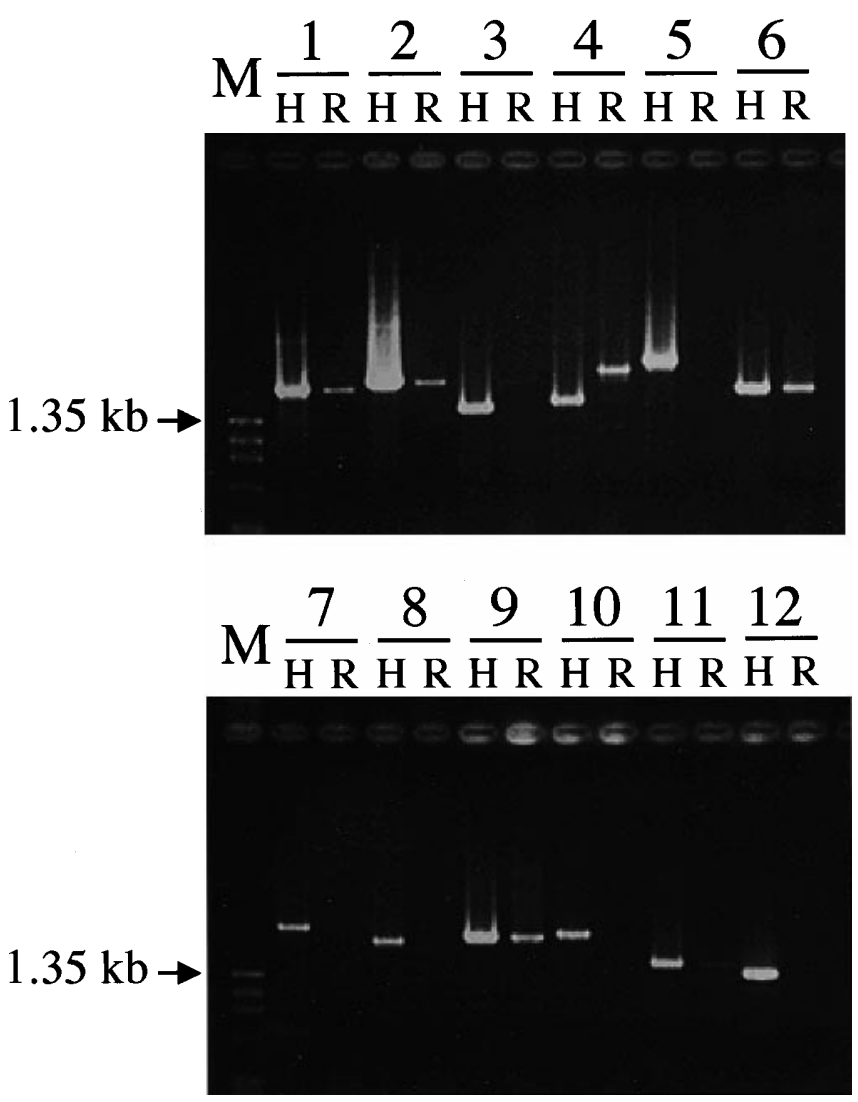

Fig. 1. Agarose gel electrophoresis of 12 fragments derived from $\mathrm{HeLa}$ and rho- 0 cells. $H$, Samples amplified from HeLa cells; $R$, samples from rho-0 cells. M shows DNA size markers (HaeIII-digested $\Phi$ X174 phage DNA). Lane 1, fragment 1, 1902 bp (nt 597-2498); lane 2, fragment 2, 2055 bp (nt 1908-3962); lane 3, fragment 3, 1517 bp (nt 3454-4970); lane 4, fragment 4, 1641 bp (nt 4659-6299); lane 5, fragment 5, 2473 bp (nt 5874-8310); lane 6, fragment 6, 1832 bp (nt 7811-9642); lane 7, fragment 7, 2335 bp (nt 9001-11,335); lane 8, fragment 8, 2025 bp (nt 10,66012,684); lane 9, fragment 9, 2137 bp (nt 12,315-14,451); lane 10, fragment 10, 2233 bp (nt 13,595-15,825); lane 11, fragment 11, 1646 bp (nt 15,071-145); and lane 12, fragment 12, 1468 bp (nt 16,134-1032) 
having almost the same lengths as those of the positive controls were detected in fragments $1,2,6$, and 9, whereas a longer product was found in fragment 4. As reported previously, fragment 2 (nt 1908-3943) was located in the region of tRNA-Leu (UUR), indicating that the presence of the nuclear pseudogene may affect the results of genetic analysis. Sequencing of fragment 2 from the rho- 0 cells showed nuclear sequences similar to those of mitochondrial tRNA-Leu (UUR). They had eight different substitutions simultaneously, five of which were the same as those reported, and three which were novel polymorphisms (Table

Table 1. Mitochondrial DNA-like sequences in the region of tRNALeu (UUR)

\begin{tabular}{ll}
\hline This study & Reported polymorphism \\
\hline G3248A & G3248A \\
T3250C & T3250C \\
A3251G & \\
C3254T & C3254T \\
A3268C & C3263T \\
C3275T & \\
G3277A & G3277A \\
T3290C & T3278C \\
& T3290C \\
\hline
\end{tabular}

(A)
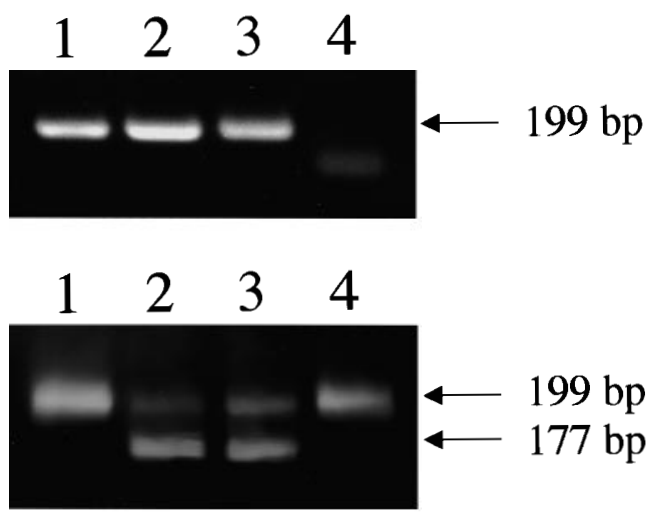

(B)

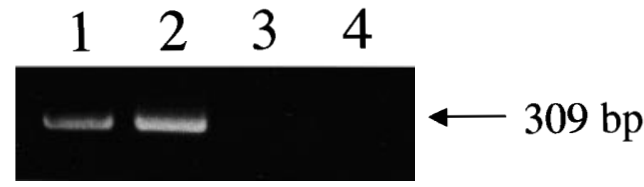

Fig. 2A,B. Detection of T3250C and T3291C substitutions. A T3250C detection. Upper panel, Agarose gel polymerase chain reaction (PCR) electrophoresis; lower panel, electrophoresis after restriction enzyme digestion. Lane 1, Normal control; lane 2, patient (positive control); lane 3, rho-0 cells; lane 4, negative control (upper) and undigested sample (lower). Note the amplified DNA band of the rho- 0 cells and the 177-bp digests. B T3291C detection. Lane 1, Normal control; lane 2, patient (positive control); lane 3, rho-0 cells; lane 4, negative control
1). A T3250C substitution also was present in our rho-0 cells, but no T3291C substitution was found.

\section{Verification of the T3250C and T3291C mutations}

Each mutation in the patients, and the rho-0 cells was examined to determine whether coamplification of the pseudogene affected the results of restriction fragment length polymorphism (RFLP) detection of T3250C and T3291C. The primers used to detect T3250C amplified the DNA from both the patients, and the rho-0 cells (Fig. 2A), whereas those for T3291C did not (Fig. 2B). Long PCR before the cycle sequencing reaction was used to avoid the effect of the pseudogene and to verify the two mutations. Whole mitochondrial DNA (16,569bp) was divided into two overlapping fragments $(6.5$ and $11.2 \mathrm{~kb})$ that could amplify the DNA from HeLa cells (Fig. 3A). No PCR products

(A)

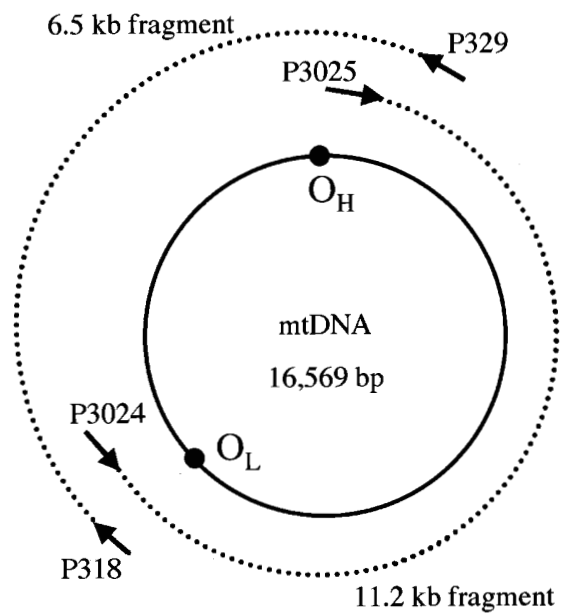

(B)

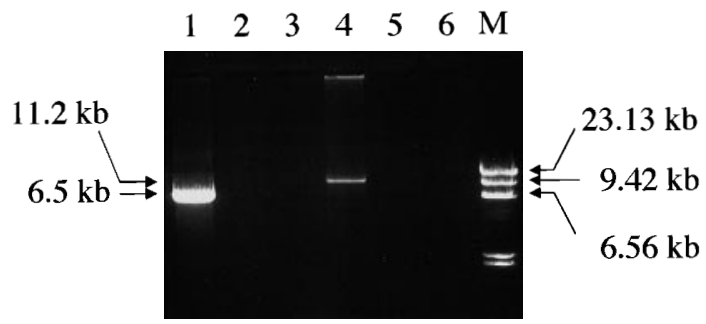

Fig. 3A,B. Long PCR for amplification of mtDNA. A Schematic representation of human mitochondrial $(m t)$ DNA and localization of each primer. The inner circle indicates mtDNA; $O_{H}$ shows replication origins of $\mathrm{H}$ strand synthesis and $O_{L}$ shows replication origins of $\mathrm{L}$ strand synthesis. The length of fragment is $6.5-\mathrm{kb}$ by P329 and P318, and 11.2kb by P3024 and P3025. B Agarose gel electrophoresis of long PCR fragments of DNA derived from HeLa and rho-0 cells. The $6.5-\mathrm{kb}$ fragments were run in lanes 1,2 , and 3 , the $11.2-\mathrm{kb}$ fragments in lanes 4, 5, and 6. Lanes 1 and 4, HeLa cells; lanes 2 and lane 5, rho-0 cells; lanes 3 and lane 6, negative control; M, DNA size marker (HindIIIdigested $\Phi$ X174 phage DNA) 
Fig. 4A,B. Nucleotide sequences around the $\mathrm{T} 3250 \mathrm{C}$ and $\mathrm{T} 3291 \mathrm{C}$ regions of the patients we reported. There is a T-to-C substitution at nucleotide $3250 \mathrm{~A}$ and a T-to-C substitution at nucleotide 3291 B

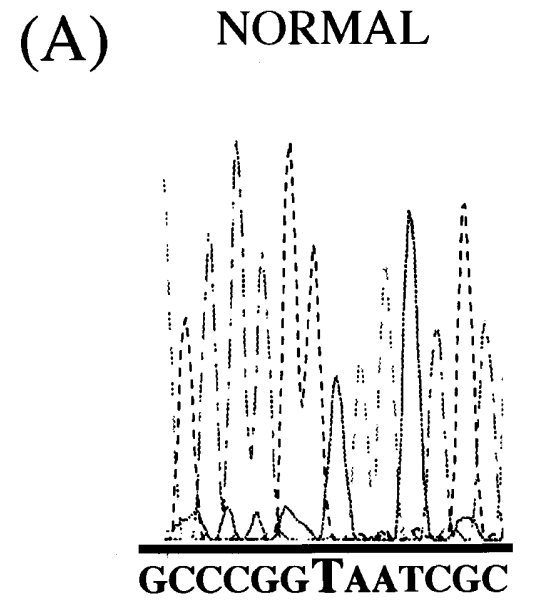

\section{PATIENT}

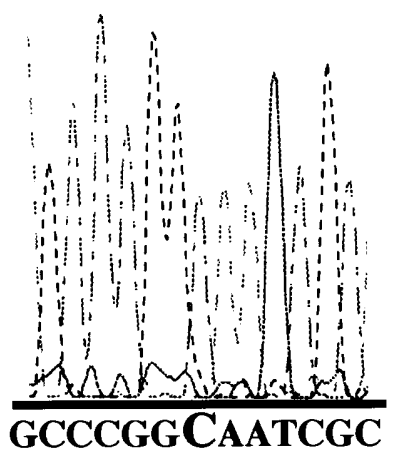

(B)

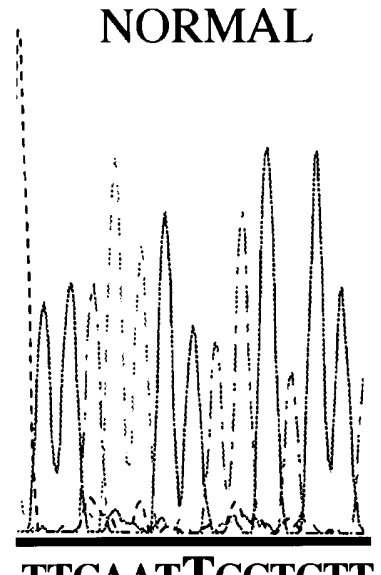

TTCAATTCCTCTT

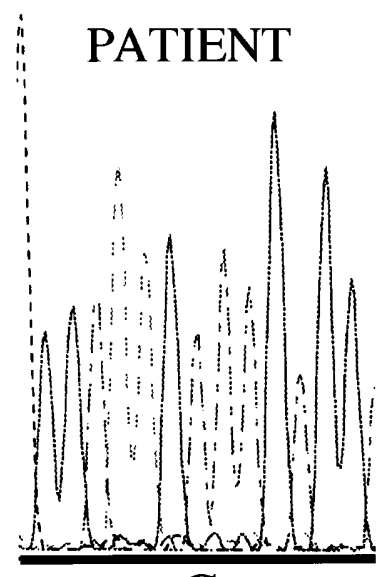

TTCAATCCCTCTT were obtained when rho-0 cell DNA was the template (Fig. 3B). Authenticity of the fragment was confirmed by sequencing analysis (data not shown). The 6.5-kb band of each patient then was extracted from the gel and sequenced. The T3250C substitution was located in the tRNA-Leu (UUR) gene. There were no polymorphisms in this region, confirming that the mutation is in the mitochondrial DNA, itself, not in the nuclear pseudogene (Fig. 4A). The T3291C mutation was compared with the published Cambridge sequence, and its location in authentic mitochondrial DNA verified (Fig. 4B).

\section{Discussion}

Most mtDNA-like sequences in the human nuclear genome are thought to be short fragments that lack contiguity and carry termination codons, frameshift deletions, and various mutations. A recent investigation found a continuous 5842bp segment of human mitochondrial DNA that spans nucleotide positions 3914 to 9755 inside the nuclear genome and is localized on chromosome 1 (Herrnstadt et al. 1999). In yeast chromosomes, the nuclear genome has several short sequences that must have originated in the mitochondrial
DNA, because the short fragment from that DNA is used to repair nuclear breakage under natural conditions (Ricchetti et al. 1999). These findings indicate that each individual has distinct pseudogenes.

The nuclear pseudogene reported here is like that noted in a previous report by Taylor et al. (1998), who reported eight polymorphisms, including T3250C and T3291C substitutions in the region. We also found eight substitutions, five of which were the same as theirs. Our rho-0 cells, however, had only a T3250C mutation in the nuclear pseudogene. We also attempted to determine the effects of the nuclear pseudogene in the genetic diagnosis of mitochondrial myopathy. A nuclear pseudogene was found by our PCRRFLP method for the detection of a T3250C substitution. This means that the nuclear pseudogene may have a harmful effect on the genetic diagnosis of most mitochondrial myopathies, because the PCR fragment lengths in various PCR-RFLP analyses usually comprise several hundreds of base pairs.

Methods used to avoid the contamination of nuclear genes are: ultracentrifugation in a cesium chloride-ethidium bromide density gradient (Clayton et al. 1967); DNA extraction from platelets which do not have a nucleus (Ito et al. 1999); and pretreatment with various restriction endonucleases before DNA extraction (Michikawa et al. 1999). 
Compared with the above-mentioned methods, the long PCR we used in this study needs no pretreatment or special extraction technique. We confirmed its usefulness in excluding the nuclear pseudogenes by no amplification using the rho-0 cell DNA as template. We think that the initial long PCR makes it possible to exclude the nuclear pseudogene and that the additional PCR produces the appropriate amount of template for sequencing, regardless of the method of extraction. Using this method, we verified the existence of the T3250C and T3291C mutations in authentic mtDNA.

Skeletal muscle has much more mtDNA than such body components as white blood cells and fibroblasts. This means that the nuclear pseudogene in the blood cells or fibroblasts may be more easily amplified than that in the skeletal muscle. Differences in the amounts of mtDNA in body tissues are associated with the amplification of the nuclear pseudogene by the PCR. It is well known that the predominant missense mutations in mitochondrial myopathy are present in higher proportions in skeletal muscle which is affected clinically, but they are usually present in lower proportions in blood cells (Rahman et al. 1999). We should be more careful to assess heteroplasmy in blood cells, because, to avoid co-amplification of the nuclear pseudogene, primers for the detection of each mutation have not yet been described.

Acknowledgments We thank Ms. Kumiko Murayama for her technical advice. This study was supported in part by research funds from the Science and Technology Agency of Japan and the Ministry of Health and Welfare of Japan (Y.G.).

\section{References}

Clayton DA, Vinograd J (1967) Circular dimer and catenate forms of mitochondrial DNA in human leukaemic leucocytes. Nature 216:652-657

Davis JN II, Parker WD (1998) Evidence that two reports of mtDNA cytochrome c oxidase "mutations" in Alzheimer's disease are based on nDNA pseudogenes of recent evolutionary origin. Biochem Biophys Res Commun 244:877-883

Davis RE, Miller S, Herrnstadt C, Ghosh SS, Fahy E, Shinobu LA, Galasko D, Thal LJ, Beal MF, Howell N, Parker WD (1997) Mutations in the mitochondrial cytochrome $\mathrm{c}$ oxidase genes segregate with late-onset Alzheimer disease. Proc Natl Acad Sci USA 94:45264531
DiMauro S, Bonilla E, Davidson M, Hirano M, Schon EA (1998) Mitochondria in neuromuscular disorders. Biochim Biophys Acta 1366:199-210

Fliss SM, Usadel H, Caballero LO, Wu L, Buta RM, Eleff MS, Jen J, Sidransky D (2000) Facile detection of mitochondrial DNA mutations in tumors and bodily fluids. Science 287:2017-2019

Goto Y, Nonaka I, Horai S (1990) A mutation in the tRNA ${ }^{\mathrm{Leu}(\mathrm{UUR})}$ gene associated with the MELAS subgroup of mitochondrial encephalomyopathies. Nature 348:651-653

Goto Y, Tojo M, Tohyama J, Horai S, Nonaka I (1992) A novel point mutation in the mitochondrial tRNA ${ }^{\text {Leu(UUR) }}$ gene in a family with mitochondrial myopathy. Ann Neurol 31:672-675

Goto Y, Tsugane K, Tanabe Y, Nonaka I, Horai S (1994) A new point mutation at nucleotide pair 3291 of the mitochondrial tRNA ${ }^{\text {Leu(UUR) }}$ gene in a patient with mitochondrial myopathy, encephalopathy, lactic acidosis, and stroke-like episodes (MELAS). Biochem Biophys Res Commun 202:1624-1630

Hayashi J-I, Ohta S, Kikuchi A, Takemitu M, Goto Y, Nonaka I (1991) Introduction of disease-related mitochondrial DNA deletions into HeLa cells lacking mitochondrial DNA results in mitochondrial dysfunction. Proc Natl Acad Sci USA 88:10614-10618

Herrnstadt C, Clevenger W, Ghosh SS, Anderson C, Fahy E, Miller S, Howell N, Davis RE (1999) A novel mitochondrial DNA-like sequence in the human nuclear genome. Genomics 60:67-77

Hirano M, Shtilbans A, Mayeux R, Davidson MM, DiMauro S, Knowles JA, Schon EA (1997) Apparent mtDNA heteroplasmy in Alzheimer disease patients and in normals due to PCR amplification of nucleus-embedded mtDNA pseudogenes. Proc Natl Acad Sci USA 94:14894-14899

Ito S, Ohta S, Nishimaki K, Kagawa Y, Soma R, Kuno S, Komatsuzaki Y, Mizusawa H, Hayashi J-I (1999) Functional integrity of mitochondrial genomes in human platelets and autopsied brain tissues from elderly patients with Alzheimer's disease. Proc Natl Acad Sci USA 96:2099-2103

Michikawa Y, Mazzucchelli F, Bresolin N, Scarlato G, Attardi G (1999) Aging-dependent large accumulation of point mutations in the human mtDNA control region for replication. Science 286:774779

Nishino I, Seki A, Maegaki Y, Takeshita K, Horai S, Nonaka I, Goto Y (1996) A novel mutation in the mitochondrial tRNA ${ }^{\text {Thr }}$ gene associated with a mitochondrial encephalomyopathy. Biochem Biophys Res Commun 225:180-185

Parfait B, Rustin P, Munnich A, Rötig A (1998) Coamplification of nuclear pseudogenes and assessment of heteroplasmy of mitochondrial mutations. Biochem Biophys Res Commun 247:57-59

Rahman S, Taanman J-W, Cooper M, Nelson I, Hargreaves I, Meunier B, Hanna MG, Garcia JJ, Capaldi RA, Lake BD, Leonard JV, Schapira AHV (1999) A missense mutation of cytochrome oxidase subunit II causes defective assembly and myopathy. Am J Hum Genet 65:1030-1039

Ricchetti M, Fairhead C, Dujon B (1999) Mitochondrial DNA repairs doublestrand breaks in yeast chromosomes. Nature 402:96-100

Taylor RW, Taylor GA, Morris CM, Edwardson JM, Turnbull DM (1998) Diagnosis of mitochondrial disease: assessment of mitochondrial DNA heteroplasmy in blood. Biochem Biophys Res Commun 251:883-887 